Original paper

\title{
Influence of track structure and condensed history physics models of Geant4 to nanoscale electron transport in liquid water
}

\author{
I. Kyriakou ${ }^{\mathrm{a}}$, V. Ivanchenko ${ }^{\mathrm{b}, \mathrm{c}}$, D. Sakata ${ }^{\mathrm{d}}$, M.C. Bordage ${ }^{\mathrm{e}, \mathrm{f}}$, S. Guatelli ${ }^{\mathrm{d}}$, S. Incerti ${ }^{\mathrm{g}, \mathrm{h}}$, \\ D. Emfietzoglou ${ }^{\text {a,* }}$
}

a Medical Physics Laboratory, University of Ioannina Medical School, Ioannina, Greece

${ }^{\mathrm{b}}$ Geant4 Associates International Ltd, Hebden Bridge, United Kingdom

${ }^{\mathrm{c}}$ Tomsk State University, Tomsk, Russia

${ }^{\mathrm{d}}$ Centre for Medical Radiation Physics, University of Wollongong, Wollongong, Australia

${ }^{\mathrm{e}}$ INSERM, UMR1037 CRCT, Université Paul Sabatier, Toulouse, France

${ }^{\mathrm{f}}$ Université Toulouse III-Paul Sabatier, UMR1037 CRCT, France

${ }^{\mathrm{g}}$ Université de Bordeaux, CENBG, Gradignan, France

${ }^{\text {h }}$ CNRS, IN2P3, CENBG, Gradignan, France

\section{A R T I C L E I N F O}

\section{Keywords:}

Geant4

Geant4-DNA

Microdosimetry

Liquid water

\begin{abstract}
A B S T R A C T
The Geant4 toolkit offers a range of electromagnetic (EM) models for simulating the transport of charged particles down to sub-keV energies. They can be divided to condensed-history $(\mathrm{CH})$ models (like the Livermore and Penelope models) and the track-structure (TS) models included in the Geant4-DNA low-energy extension of Geant4. Although TS models are considered the state-of-the-art for nanoscale electron transport, they are difficult to develop, computationally intensive, and commonly tailored to a single medium (e.g., water) which prohibits their use in a wide range of applications. Thus, the use of $\mathrm{CH}$ models down to sub-keV energies is particularly intriguing in the context of general-purpose Monte Carlo codes. The aim of the present work is to compare the performance of the $\mathrm{CH}$ models of Geant4 against the recently implemented TS models of Geant4DNA for nanoscale electron transport. Calculations are presented for two fundamental quantities, the dose-pointkernel and the microdosimetric lineal energy. The influence of user-defined simulation parameters (tracking and production cuts, and maximum step size) on the above calculations is also examined. It is shown that Livermore offers the best performance among the $\mathrm{CH}$ models of Geant4 for nanoscale electron transport. However, even under optimally-chosen simulation parameters, the differences between the $\mathrm{CH}$ and TS models examined may be sizeable for low energy electrons $(<1 \mathrm{keV})$ and/or nanometer size targets $(<100 \mathrm{~nm})$.
\end{abstract}

\section{Introduction}

It is well-established that cellular radiobiological effects mainly originate from energy deposition at the nanoscale which is associated with important subcellular structures (e.g., DNA) [1,2]. At this spatial scale the transport of low-energy electrons, loosely defined as those with kinetic energy below $\sim 1-10 \mathrm{keV}$, becomes a critical component of any quantitative analysis of radiation effects because their penetration in matter (here soft tissue) is comparable to the target dimensions $(<1$ $\mu \mathrm{m})$. Thus, low-energy electrons have traditionally been one of the primary subject of study in microdosimetry and radiobiological modeling $[3,4]$.

Monte Carlo track structure (TS) models are considered the state-ofthe-art for nanoscale electron transport because they enable simulation of individual interactions (analogue transport) down to $\sim \mathrm{eV}$ energies [5]. At the heart of TS models are the interaction cross sections of all the physical process included in the simulation (e.g., shell ionization, electronic excitation, elastic scattering). Over the past decades several TS codes have been developed in the context of microdosimetry and radiobiological modeling of cellular effects, e.g., NOREC [6], PARTRAC [7], KURBUC [8], RETRACKS (RITRACKS) [9], Geant4-DNA [10], among others [11]. The main problem with TS models is that they contain a high-degree of detail and, therefore, they are difficult to develop [12]. As a result, TS models are commonly tailored to a single medium (e.g., water) which prohibits their use in general-purpose codes [13]. Finally, TS simulations are computationally intensive so they are commonly restricted to low energies, e.g., electrons below $\sim 100 \mathrm{keV}[14]$.

\footnotetext{
* Corresponding author.

E-mail address: demfietz@gmail.com (D. Emfietzoglou).
} 
The opposite to TS models are the condensed-history (CH) models which aim to account for the cumulative effect of a large numbers of interactions ( $>10-20$ ) along a small segment of the track (called step). At the heart of $\mathrm{CH}$ models are multiple-scattering theories for the energy-loss, angular deflection, and spatial diffusion of the particle along the step [15]. Because of their computational efficiency $\mathrm{CH}$ models are presently the method of choice for the transport of high-energy electrons ( $>1 \mathrm{MeV}$ ) and represent the gold-standard in macroscopic clinical dosimetry [16]. Moreover, by simulating a subset of collisions (referred to as hard or catastrophic) in an analogue manner through single-scattering cross sections, $\mathrm{CH}$ models may allow for secondary particle production ( $\delta$-rays, bremsstrahlung photons), thus, improving their dosimetric resolution [17]. Several $\mathrm{CH}$ codes are available today for electron (and other charged particle) transport, perhaps the most known being EGS [18], MCNP [19], PENELOPE [20], FLUKA [21], and Geant4 [22-24]. The Geant4 toolkit offers several $\mathrm{CH}$ models for electron transport down to very low energies, although the level of accuracy of those models at sub-keV energies is substantially reduced. For the PENELOPE code [20] the accuracy of the low-energy models (e.g., down to $\sim 100 \mathrm{eV}$ or even lower) has been studied [25]. The possibility of using $\mathrm{CH}$ models for sub-keV electron transport is intriguing given that, compared to TS models, they can be easily applied to different materials and simulations are much faster. Nevertheless, systematic studies of the low energy performance of $\mathrm{CH}$ models are limited [26-28].

The aim of the present work was to compare the performance of the CH models of Geant4 (Livermore, Penelope, Standard) against the TS models recently implemented in Geant4-DNA for nanoscale electron transport. Benchmark calculations are presented for two fundamental quantities in dosimetry and microdosimetry, namely, the dose-pointkernel and the lineal energy, respectively. The influence of user-defined simulation parameters (tracking and production cuts, and maximum step size) on the above calculations is also examined. While the present work is a comparison between Monte Carlo models, thus lacking the added value of a validation with experimental data, it is of potential interest for a large community of Geant4 users. Employing a $\mathrm{CH}$ model instead of a TS model, besides the ease of application to many different materials, generally results in a significant speed-up in terms of CPU and, therefore, could be a fair trade-off, if the relative precision is specified.

\section{Method}

\subsection{Geant4 electromagnetic (EM) physics}

An alternative to the Standard Electromagnetic (EM) models of Geant4 is the Low-Energy EM models category. The latter includes both $\mathrm{CH}$ and TS models [28]. The Low-Energy CH models come in two flavors, the "Livermore" and "Penelope" models which are available to Geant4 users through the "G4EmLivermorePhysics" and "G4EmPenelopePhysics" constructors, respectively. As regards electrons, both models offer a mixed simulation approach which combines multiplescattering theories for elastic collisions and soft energy losses and single-scattering cross sections for hard inelastic collisions (e.g., $\delta$-ray production). In this approach, the user must also define the energy threshold for the production of secondary particles (i.e., delta electrons and bremsstrahlung photons), the so-called "production cut". The role of the production cut is to distinguish between soft and hard inelastic collisions which are treated in a continuous and discrete manner, respectively. The TS models are contained in the Geant4-DNA low-energy extension of Geant4. Three recommended choices are available to users which are often referred to as the "default" [29], "Ioannina" [30], and "CPA100" [31] models. These are fully discrete models available through the "G4EmDNAPhysics_option2" (or DNA option2), "G4EmDNAPhysics_option4" (or DNA option4), and "G4EmDNAPhysics_option6" (or DNA option6) constructors, respectively.
In both the $\mathrm{CH}$ and TS models the user must also define the energy threshold for particle absorption; the so-called "tracking cut". This is the energy value below which the charged particles are no longer propagated (i.e., they are "killed") and all their energy is deposited locally.

\subsection{Geant4-Livermore}

This EM physics constructor is intended for the transport of electrons and photons down to low energies in elemental materials with $\mathrm{Z}=1-100$. Livermore physics is based on the EPDL97 [32], EADL [33], and EEDL [34] public data libraries. Electron-impact ionization is calculated theoretically according to Seltzer's prescription which combines a modified Weizsacker-Williams expression for soft collisions and a modified Möller formula for hard collisions [35]. Both modifications take into account the binding of atomic electrons to a given subshell. Excitations are approximated as soft ionizations using the contribution of the outermost subshell [34]. By taking into account the shell structure of atomic elements, Livermore is also able to simulate atomic deexcitation processes from most of shells, such as fluorescence and Auger cascades. In the Geant4-Livermore physics constructor multiple elastic scattering is not based on the EEDL elastic scattering cross sections but is simulated using the Geant4 Urban model [36]. The low energy limit of the Livermore ionization model is $10 \mathrm{eV}$ although reduced accuracy is expected below $\sim 100 \mathrm{eV}$ [34]. Originally, the Livermore models were recommended for electron energies above $250 \mathrm{eV}$ (which includes the K-shell of elements down to carbon, $Z=6$ ) [37]. In the present context it is important to highlight that Livermore physics disregards solid-state effects.

\subsection{Geant4-Penelope}

This EM constructor is also intended for the transport of low-energy electrons, positrons, and photons in elemental materials with $\mathrm{Z}=1-100$. The Penelope physics is based on a re-engineering of the PENELOPE code version 2008. Electron-impact ionization is calculated within the first Born approximation based on the Generalised Oscillator Strength (GOS) model of Liljequist [38] which distinguishes between soft and hard collisions using delta functions. According to this model the contribution of excitations is ignored while the outershell electronic structure is accounted for very roughly [25]. However, the analytic properties of this GOS model allow fast random sampling of energy-loss and $\delta$-rays [13]. Atomic de-excitation processes are considered only for $\mathrm{K}, \mathrm{L}$, and M shells. As in Livermore, multiple elastic scattering in the Geant4-Penelope constructor is simulated using the Geant4 Urban model [36]. The low energy limit of the Penelope models is $100 \mathrm{eV}$ [20]. In principle, the low energy limit can be set as low as $10 \mathrm{eV}$, although the production cut cannot be set lower than $100 \mathrm{eV}$. As in Livermore, solid-state effects are also disregarded in the Penelope physics.

\subsection{Geant4 standard option4}

Calculations are also made with the Standard option4 constructor which is designed as a collection of the most accurate Geant4 EM models for each particle type and energy range. For the Geant 4 version 10.4 in the low-energy domain $(<1 \mathrm{MeV})$, the Standard option4 constructor employs the Penelope ionization model and the GoudsmitSaunderson multiple scattering model for elastic collisions. Although the default low energy limit of the Standard option4 constructor is $100 \mathrm{eV}$, it can also be extended down to $10 \mathrm{eV}$ (under the restrictions of the Penelope models mentioned earlier).

\subsection{Geant4-DNA}

Contrary to the above mentioned $\mathrm{CH}$ models, the Geant4-DNA 
package includes TS models that enable analog simulation of chargedparticle transport in liquid water medium down to $\mathrm{eV}$ energies $(\sim 10 \mathrm{eV})[39]$. Regarding the simulation of electron interactions, users may select among three recommended sets of alternative physics models (constructors), namely, the "default" (or DNA option2), the "Ioannina" (or DNA option4), and the "CPA100" (or DNA option6). These sets of models correspond to different cross sections for elastic and inelastic scattering. The default and Ioannina models, which cover the energy range $7.4 \mathrm{eV}-1 \mathrm{MeV}$ and $10 \mathrm{eV}-10 \mathrm{keV}$, respectively, are methodologically similar and use the energy-loss-function (ELF) formalism. The Ioannina models essentially improve the ionization and excitation cross sections of the default models, effective at sub-keV electron energies $[13,39]$. In both models, ionization and excitation cross sections are calculated in the Born approximation using the ELF parameterization of Emfietzoglou and co-workers [40-43] which is based on experimental data for liquid water. Thus, solid-state effects are automatically included via the properties of the ELF. Elastic cross sections are treated by the partial wave method (default) or by an empirical screened Rutherford formula (Ioannina).

In CPA100 [31] the ionization cross sections are calculated from the binary-encounter-Bethe (BEB) atomic model. This has the advantage that total and differential ionization cross sections are calculated analytically to the expense that solid-state effects are disregarded. Such effects are included only in the excitation cross sections which are calculated by the ELF parameterization of Dingfelder and co-workers [44]. The use of different methods to calculate inelastic cross sections (BEB for ionizations and ELF for excitations) compromises the selfconsistency of this approach. Elastic scattering cross sections are based on partial wave calculations using the independent atom approximation.

\subsection{Simulations}

All simulations are based on Geant 4 version 10.4. The number of histories used for the calculations were between $10^{6}$ to $10^{7}$ with the resulting statistical uncertainty being below $1 \%$. Calculations are presented for the dose-point-kernel (DPK) and the lineal energy using, respectively, the "TestEm12" and "microyz" examples [39]. In Geant4, an example is a ready-to-use application which is provided with its source code distribution, including macro files containing Geant4 commands that enable an easy control of the simulation parameters without the need for recompilation of the user application. The results presented in this work have been produced exclusively by the above examples (dna.mac and microyz.in are the respective macro files for the "TestEm12" and "microyz" examples).

The results of the Geant4 $\mathrm{CH}$ models (Livermore, Penelope, Standard Option4) are compared against the recently implemented Geant4-DNA TS models (Ioannina or option4) which are used as reference.

DPK quantifies the dose distribution around a point isotropic source [45] and it is a widely used reference parameter in radiopharmaceutical dosimetry [46]. DPK calculations for monoenergetic electrons $(100 \mathrm{eV}-10 \mathrm{keV})$ are based on the "TestEm12" example using a fixed bin size (concentric shell) of $1 \mathrm{~nm}$ width which is roughly the spatial resolution of the TS simulations used as reference.

The lineal energy, on the other hand, is a well-established parameter in microdosimetry [47] and widely used to represent the quality of the radiation [48]. Lineal energy calculations for monoenergetic electrons $(100 \mathrm{eV}-10 \mathrm{keV})$ are based on the "microyz" example using a 10 and $100 \mathrm{~nm}$ sphere diameter. This is roughly the range of target sizes that have been found to correlate with clinical RBE values in radiation therapy [49] while also associated with biophysical models of radiation action [50].

In Geant4, the step size of a charged particle dynamically depends on particle type, energy, material properties, and user cuts in range $[22,24]$. However, the Geant 4 user may force the maximum step size value (called step size limit) of all simulation steps using the so-called "G4StepLimiter" process. Similarly, the tracking and production cuts which determine the energy thresholds for particle kill and particle production, respectively, may be set by the user. The influence of the above user-defined simulation parameters will also be examined in the present study. Specifically, values of the step size limit (SL) between 0.1 and $100 \mathrm{~nm}$ and tracking and production cuts between 10 and $100 \mathrm{eV}$ were studied.

\section{Results and discussion}

\subsection{DPK profiles}

The comparison of the DPK profiles is based on the relative bin-bybin difference defined by the expression:

$\Delta_{\text {bin }}(\%)=\frac{\left|\mathrm{DPK}_{\text {model }}\left(r_{\text {bin }}\right)-\mathrm{DPK}_{\text {DNAopt } 4}\left(r_{\text {bin }}\right)\right|}{\operatorname{Max}\left\{\mathrm{DPK}_{\text {DNAopt } 4}\right\}} \times 100$

where $\mathrm{DPK}_{\text {model }}\left(r_{\text {bin }}\right)$ is the value of the DPK profile at distance $r_{\text {bin }}$ from the emission point calculated by a particular $\mathrm{CH}$ set of models (here Livermore, Penelope, or Standard option4). Similarly, $\mathrm{DPK}_{\mathrm{DNAopt}}\left(r_{\text {bin }}\right)$ is the corresponding value calculated from the "DNA option4" (or Ioannina) set of TS models. Eq. (1) is normalised to the maximum of the $\mathrm{DPK}_{\mathrm{DNAopt} 4}$ profile in order to avoid the effect of large fluctuations at large $r_{\text {bin }}$ associated with small dose values that are not of practical interest. To reduce the comparison to a single index for each DPK profile (as in [51]), we have plotted the maximum value of Eq. (1), $\Delta_{\max }(\%)=\operatorname{Max}\left\{\Delta_{\text {bin }}(\%)\right\}$.

In Fig. 1a, 1b, and 1c we present the $\Delta_{\max }(\%)$ for the $\mathrm{CH}$ models and for the different SL examined (tracking and production cuts $=10 \mathrm{eV}$ ). Penelope exhibits the largest differences from DNA option4 whereas Livermore has the best agreement with DNA option4, in agreement with recent findings $[27,28]$. Livermore DPK calculations also seem to be the least sensitive to SL variations. For all $\mathrm{CH}$ models, the agreement with the TS models becomes better with increasing electron energy and for smaller SL $(0.1 \mathrm{~nm}$ or $1 \mathrm{~nm})$.

The effect of the tracking and production cuts is presented in Fig. 2. Clearly, there is an increase of $\Delta_{\max }(\%)$ as the tracking and production cuts are raised from $10 \mathrm{eV}$ (used as reference) to $50 \mathrm{eV}$ and $100 \mathrm{eV}$. Overall, the effect is small $(<5 \%)$ for electrons with initial energy above $1 \mathrm{keV}$, but sizeable (up to $\sim 35 \%$ ) for sub-keV energies. Among the $\mathrm{CH}$ models, Livermore DPK calculations are the most sensitive to the variations of the tracking and production cuts between 10 and $100 \mathrm{eV}$. This is because in the Penelope models (and Standard option 4 models which use Penelope ionization), the production cut cannot fall below $100 \mathrm{eV}$ due to a default internal setting in Geant4. Thus, in the sub$100 \mathrm{eV}$ energy range, the Penelope (and Standard option4) models is only affected by the lowering of the tracking cut which renders this model somewhat less sensitive than Livermore (which is affected by the lowering of both the tracking and production cut).

\subsection{Microdosimetry profiles}

The comparison of the lineal energy profiles is based on the expression:

$\Delta_{\text {rel }}(\%)=\left|\frac{\bar{y}_{\text {model }}(T)}{\bar{y}_{\text {DNAopt } 4}(T)}-1\right| \times 100$

where $\bar{y}_{\text {model }}(T)$ and $\bar{y}_{\text {DNAopt } 4}(T)$ are the values of the frequency-mean lineal energy at electron energy $\mathrm{T}$ for a particular $\mathrm{CH}$ set of models (here Livermore, Penelope, or Standard option4) and the "DNA option4" (or Ioannina) set of TS models, respectively. To reduce the comparison to a single index, we have also used the maximum value of Eq. (2), $\Delta_{\max }(\%)=\operatorname{Max}\left\{\Delta_{\text {rel }}(\%)\right\}$.

In Figs. 3a, b, c and 4a, b, c we present the $\Delta_{\text {rel }}(\%)$ for the $\mathrm{CH}$ models 

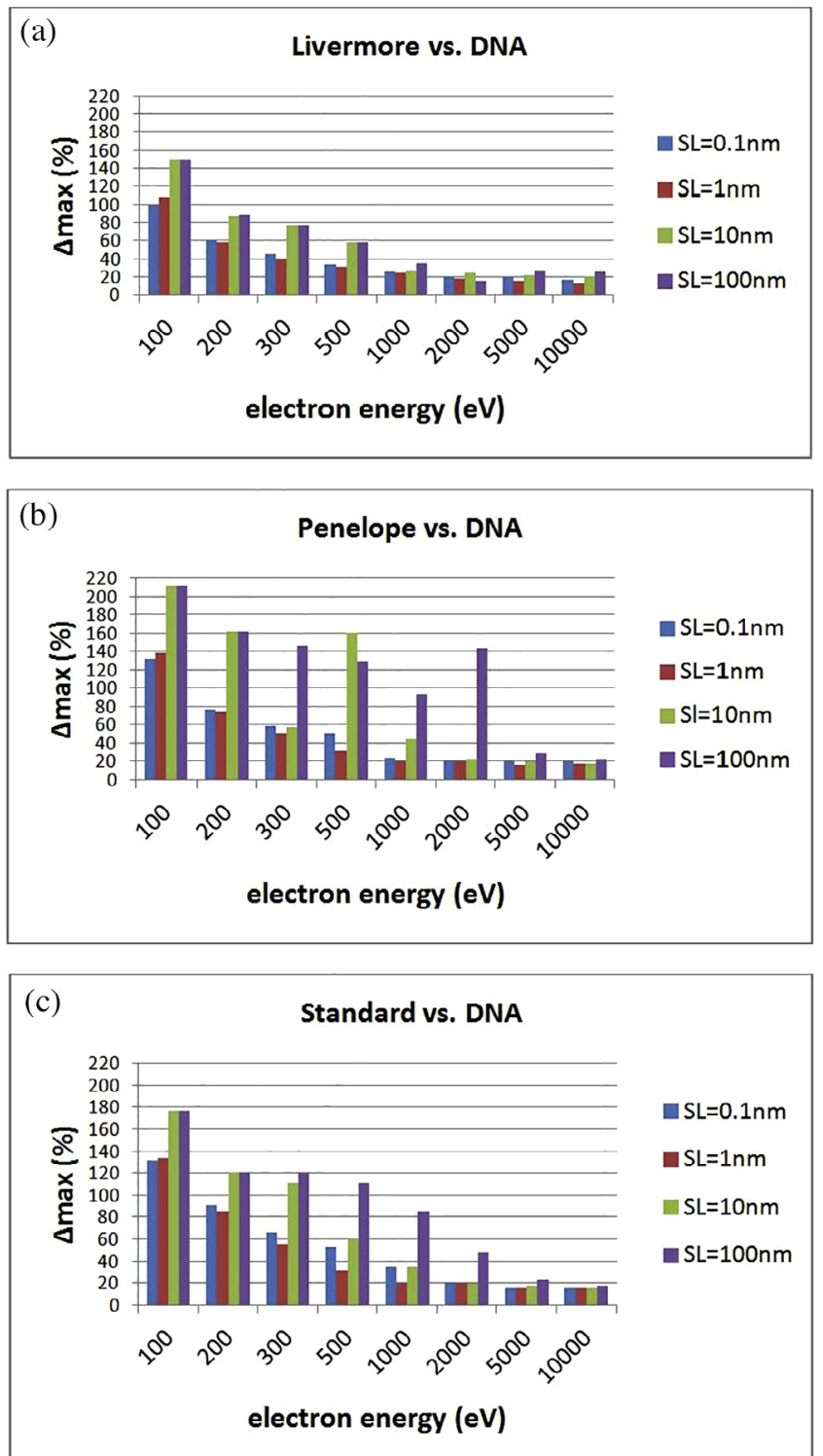

Fig. 1. The maximum DPK difference, $\Delta_{\max }(\%)$, as a function of initial electron kinetic energy for each $\mathrm{CH}$ model and SL value. The tracking and production cuts are set equal to $10 \mathrm{eV}$. The Geant4-DNA option 4 model is used as reference.
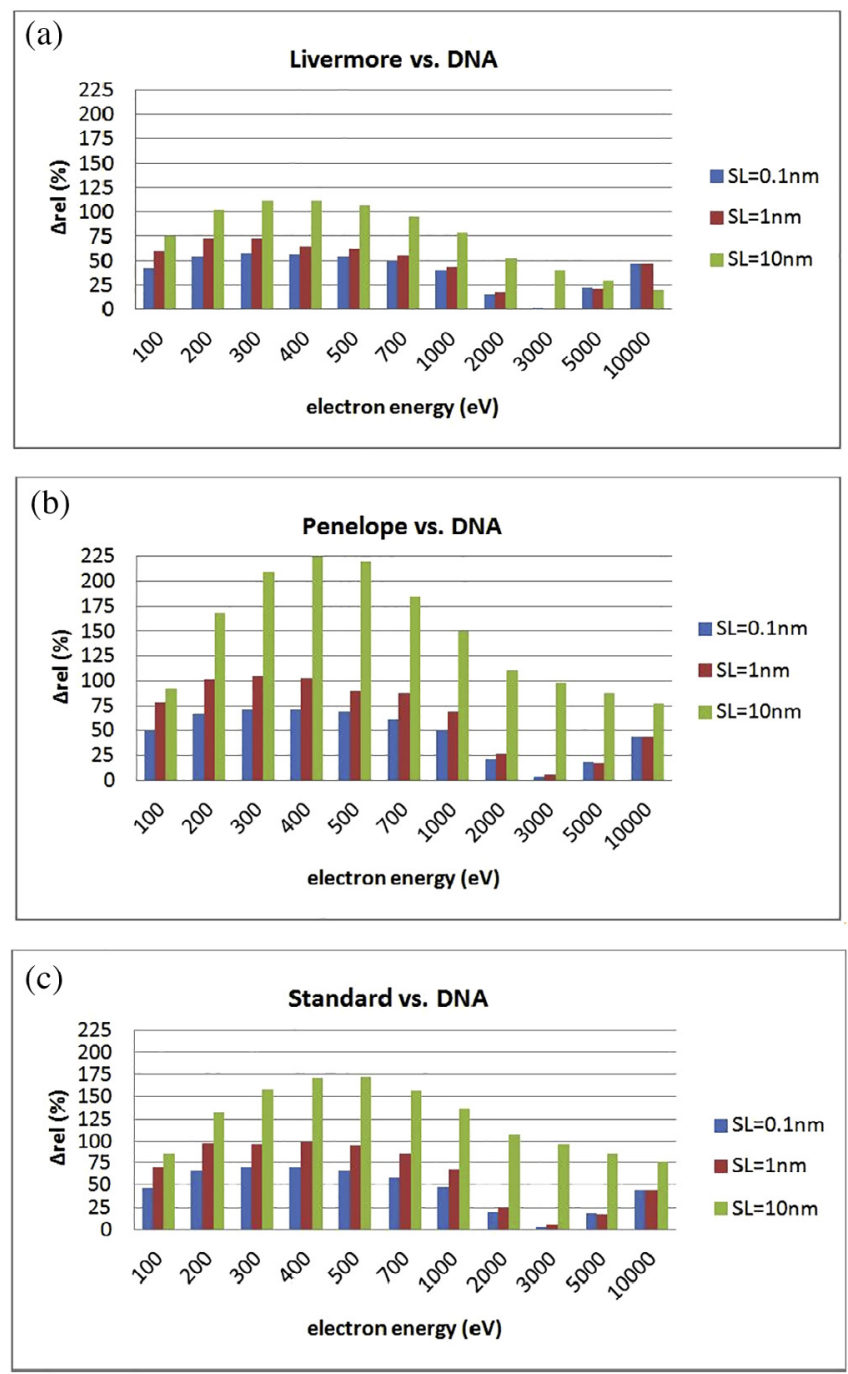

Fig. 3. The frequency-mean lineal energy difference, $\Delta_{\text {rel }}(\%)$, as a function of initial electron kinetic energy for each $\mathrm{CH}$ model and SL value for a target sphere of $10 \mathrm{~nm}$ diameter. The tracking and production cuts are set equal to $10 \mathrm{eV}$. The Geant4-DNA option4 model is used as reference.

and the different SL values examined for target spheres of $10 \mathrm{~nm}$ and $100 \mathrm{~nm}$ diameter, respectively. Note that we have only used SL values that satisfy the condition $\mathrm{SL}_{\max } \leq$ diameter. The tracking and

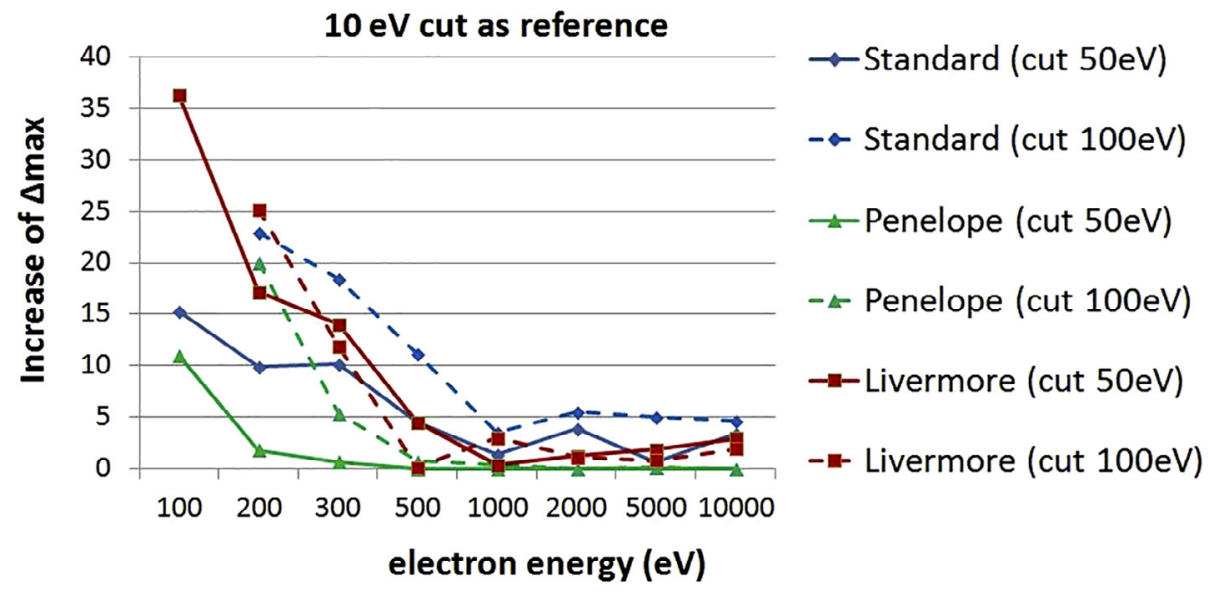

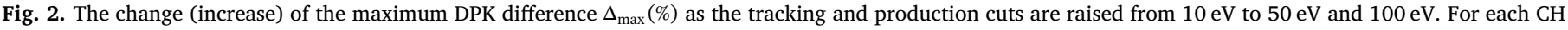
model, the value of $\Delta_{\max }(\%)$ for tracking and production cut equal to $10 \mathrm{eV}$ is used as reference. 

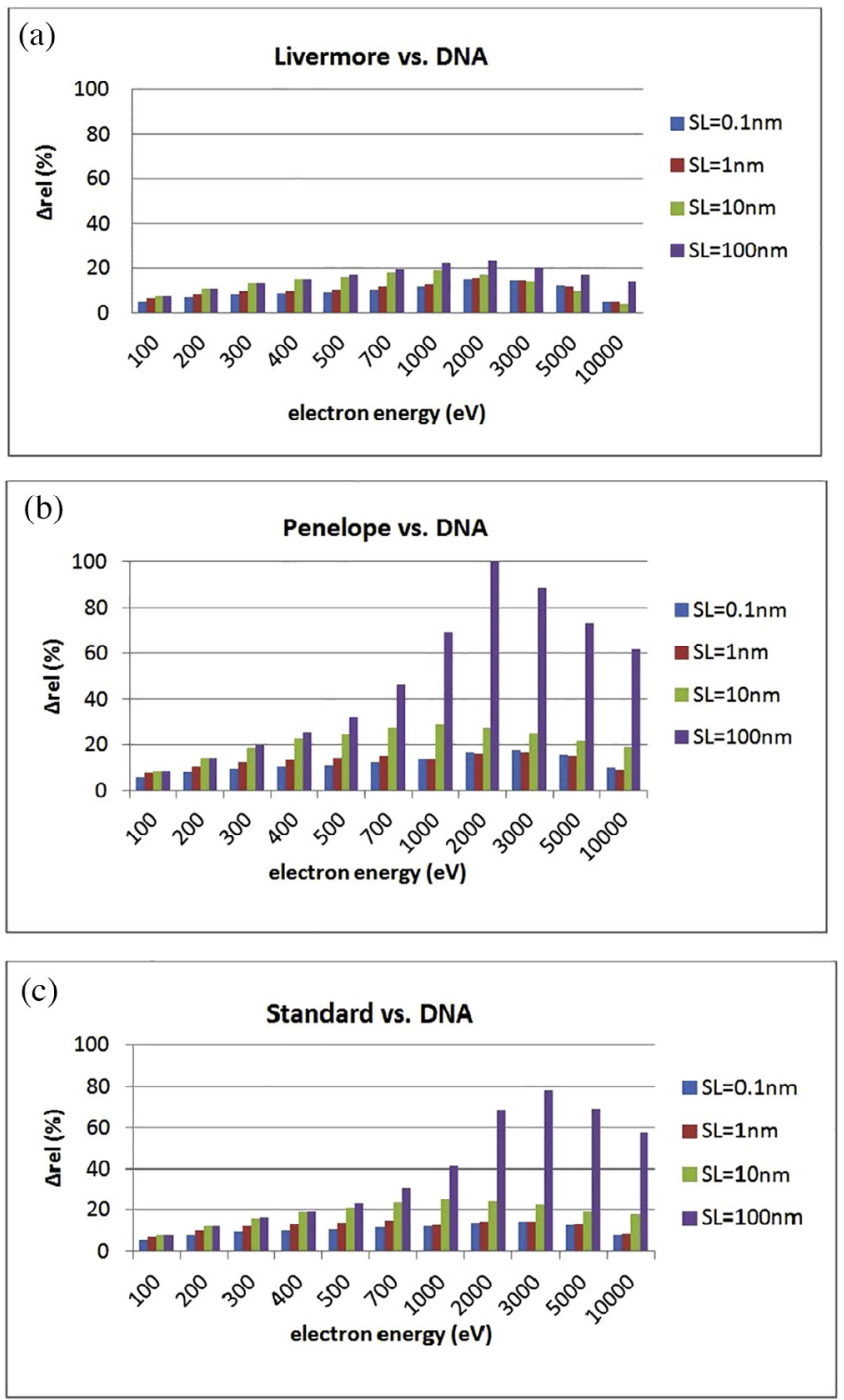

Fig. 4. The frequency-mean lineal energy difference, $\Delta_{\text {rel }}(\%)$, as a function of initial electron kinetic energy for each $\mathrm{CH}$ model and SL value for a target sphere of $100 \mathrm{~nm}$ diameter. The tracking and production cuts are set equal to $10 \mathrm{eV}$. The Geant4-DNA option4 model is used as reference. production cuts were set equal to $10 \mathrm{eV}$ for these simulations. The performance of the models is similar to what we have observed for the DPK. Overall, Penelope has the largest differences from DNA option4 whereas Livermore exhibits the best agreement with DNA option4. Livermore lineal energy calculations also seem to be the least sensitive to SL variations. For all $\mathrm{CH}$ models, best agreement with the TS models is obtained for the smallest SL $(0.1 \mathrm{~nm})$. Based on the simulation results with the smallest SL $(0.1 \mathrm{~nm})$, the differences between the $\mathrm{CH}$ and TS models are significant for the $10 \mathrm{~nm}$ sphere (up to $\sim 60 \%$ for Livermore and $\sim 70 \%$ for Penelope and Standard option 4 ), but only moderate for the $100 \mathrm{~nm}$ sphere (up to $\sim 15 \%$ for all models). Note that, contrary to the DPK, the agreement between $\mathrm{CH}$ and TS models for the lineal energy depens upon both the electron energy and the sphere diameter. A similar conclusion was drawn by Lazarakis et al. [27] using a more simplified irradiation geometry. However, in the present study, the relationship between track length and sphere diameter is more complicated than in [27] owing to the random overlap between the electron track structure and the target (as required from the definition of lineal energy).

The effect of the tracking and production cuts is presented in Fig. 5. Clearly, raising the the tracking and production cuts from $10 \mathrm{eV}$ to $50 \mathrm{eV}$ and $100 \mathrm{eV}$ has a negligible effect $(<5 \%)$ for the $100 \mathrm{~nm}$ sphere but a sizeable effect for the $10 \mathrm{~nm}$ sphere (up to $\sim 15 \%$ for Penelope, $\sim 20 \%$ for Standard option 4 , and $\sim 40 \%$ for Livermore). Among the $\mathrm{CH}$ models, Livermore is the most sensitive to variations of the tracking and production cuts, as also found for the DPK (and explained above).

\section{Conclusion}

We have undertaken calculations of electron dose-point-kernel and lineal energy in liquid water medium using some of the $\mathrm{CH}$ and TS models of Geant4 version 10.4. The influence of some user-defined simulation parameters (tracking and production cuts and maximum step size) on the above calculations has also been examined. Using the TS models as reference, it was shown that Livermore offers the best performance among the $\mathrm{CH}$ models for nanoscale electron transport. Also, Livermore was found to be the least sensitive to SL variation among the $\mathrm{CH}$ models but the most sensitive to changes of the tracking and production cuts. However, users must be cautious because, even under optimally-chosen simulation parameters, the differences between the $\mathrm{CH}$ and TS models of Geant4 may still be significant for low energy electrons $(<1 \mathrm{keV})$ and/or nanometer size targets $(<100 \mathrm{~nm})$. The present findings are in agreement with recent studies on the performance of the low energy electromagnetic models of Geant4 [27,28] where it was concluded that simulations at the nanoscale strongly depend upon the target size, the physics model, and simulation

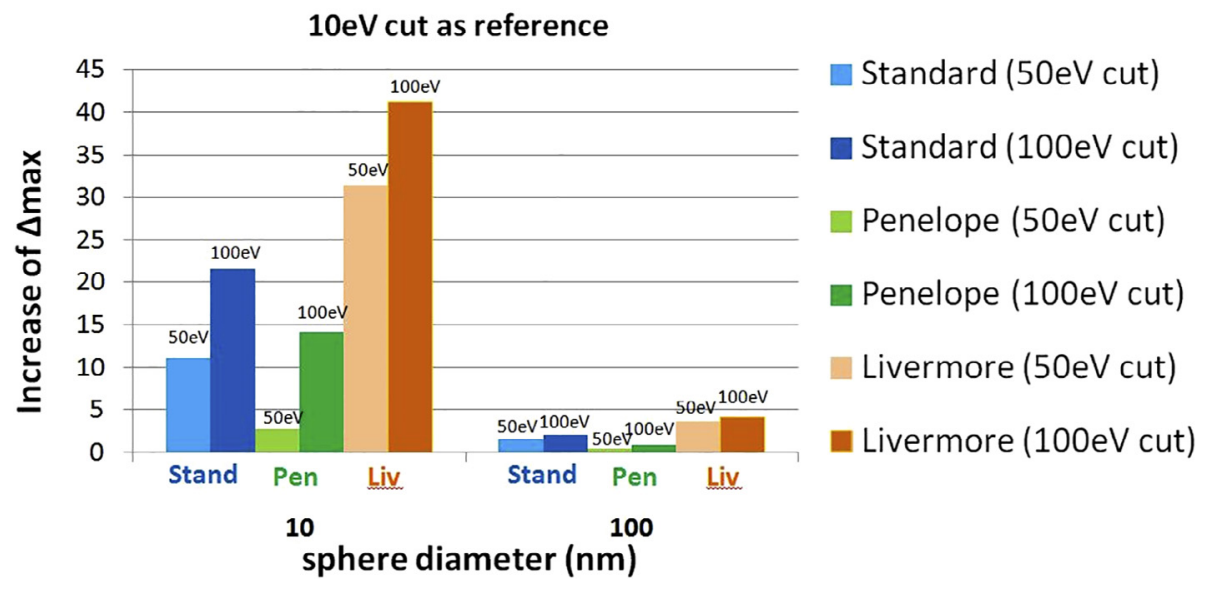

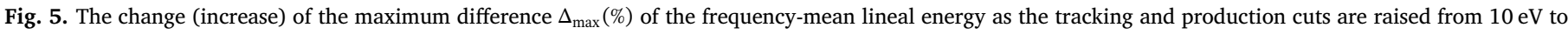
$50 \mathrm{eV}$ and $100 \mathrm{eV}$. For each $\mathrm{CH}$ model, the value of $\Delta_{\max }(\%)$ for tracking and production cut equal to $10 \mathrm{eV}$ is used as reference. 
parameters. Overall, for initial electron energies at the sub-keV range, the $\mathrm{CH}$ models may considerably overestimate the energy deposition in nanometer targets calculated by TS models. Therefore, it is important that efforts are invested to extend the application of TS models to different target materials and further reduce their uncertainties by taking advantage new experimental data and theoretical advances.

\section{Acknowledgements}

We would like to acknowledge financial support from the CNRS PICS \#7340 France - Greece and the IdEx Bordeaux University - France - International Post-doctorates program in the framework of the "France-Japan Particle Physics Laboratory" International Associated Laboratory. S. Guatelli and D. Sakata acknowledge financial support from the Australian Research Council, ARC DP170100967. I. Kyriakou and D. Emfietzoglou acknowledge financial support from ESA (Contract No. 4000112863/14/NL/HB).

\section{References}

[1] Nikitaki Z, Nikolov V, Mavragani IV, Plante I, Emfietzoglou D, Iliakis G, et al. NonDSB clustered DNA lesions. Does theory colocalize with the experiment? Radiat Phys Chem 2016;128:26-35.

[2] Nikjoo H, Taleei R, Liamsuwan T, Liljequist D, Emfietzoglou D. Perspectives in radiation biophysics: from radiation track structure simulation to mechanistic models of DNA damage and repair. Radiat Phys Chem 2016;128:3-10.

[3] Nikjoo H, Emfietzoglou D, Liamsuwan T, Taleei R, Liljequist D, Uehara S. Radiation track, DNA damage and response—a review. Rep Progr Phys 2016;79:116601.

[4] Dingfelder M. Track structure: time evolution from physics to chemistry. Radiat Prot Dosim 2006;122:16-21.

[5] Dingfelder M. Track-structure simulations for charged particles. Health Phys 2012;103:590-5.

[6] Semenenko VA, Turner JE, Borak TB. NOREC a Monte Carlo code for simulating electron tracks in liquid water. Radiat Environ Biophys 2003;42:213-7.

[7] Dingfelder M, Ritchie RH, Turner JE, Friedland W, Paretzke HG, Hamm RN. Comparisons of calculations with PARTRAC and NOREC: Transport of electrons in liquid water. Radiat Res 2008;169:584-94.

[8] Liamsuwan T, Emfietzoglou D, Uehara S, Nikjoo H. Microdosimetry of low-energy electrons. Int J Radiat Biol 2012;88:899-907.

[9] Plante I, Cucinotta FA. Cross sections for the interactions of $1 \mathrm{eV}-100 \mathrm{MeV}$ electrons in liquid water and application to Monte-Carlo simulation of HZE radiation tracks. New J Phys 2009;11:063047.

[10] Bernal MA, Bordage MC, Brown JMC, Davidkova M, Delage E, El Bitar Z, et al. Track structure modeling in liquid water: a review of the Geant4-DNA very low energy extension of the Geant4 Monte Carlo simulation toolkit. Phys Med 2015;31:861-74.

[11] Nikjoo H, Uehara S, Emfietzoglou D, Cucinotta FA. Monte Carlo track structure codes in radiation research - A review. Radiat Meas 2016;41:1052-74.

[12] Fernández-Varea JM, Llovet X, Salvat F. Cross sections for electron interactions in condensed matter. Surf Interf Anal 2005;37:824-32.

[13] Fernandez-Varea JM. Monte Carlo simulation of the inelastic scattering of electrons and positrons using optical-data models. Radiat Phys Chem 1998;53:235-45.

[14] Andreo P. Monte Carlo techniques in medical radiation physics. Phys Med Biol 1991;36:861-920.

[15] Salvat F, Fernández-Varea JM. Overview of physical interaction models for photon and electron transport used in Monte Carlo codes. Metrologia 2009;46:S112-38.

[16] Chetty IJ, Curan B, Cygler JE, DeMarco JJ, Ezzell G, Fadeggon BA, et al. Report of the AAPM Task Group No. 105: Issues associated with clinical implementation of Monte Carlo-based photon and electron external beam treatment planning. Med Phys 2007;34:4818-53.

[17] Nahum AE. Condensed-history Monte-Carlo simulation for charged particles: what can it do for us? Radiat Environ Biophys 1999;38:163-73.

[18] Kawrakow I, Mainegra-Hing E, Rogers DWO, Tessier F, Walters PRB. The EGSnrc Code System: Monte Carlo Simulation of Electron and Photon Transport. NRCC Report PIRS-701; 2017.

[19] Briesmeister JF. MCNP-A General Monte Carlo Code for Neutron and Photon Transport. LA-7396-M 3A. Los Alamos: Los Alamos National Laboratory; 1986.

[20] Baro J, Sempau J, Fernandez-Varea JM, Salvat F. PENELOPE: an algorithm for Monte Carlo simulation of the penetration and energy loss of electrons and positrons in matter. Nucl Instrum Meth B 1995;100:31-46.

[21] Ferrari A, Sala PR, Fasso A, Ranft J. FLUKA: A Multi-Particle Transport Code (Program version 2005). Geneva: CERN; 2005.

[22] Agostinelli S, Allison J, Amako K, Apostolakis J, Araujo H, Arce P, et al. Geant4 - a simulation toolkit. Nucl Instrum Meth A 2003;506:250-303.
[23] Allison J, Amako K, Apostolakis H, Araujo H, Arce P, Asai M, et al. Geant4 developments and applications. IEEE Trans Nucl Sci 2006;53:270-8.

[24] Allison J, Amako K, Apostolakis J, Arce P, Asai M, Aso T, et al. Recent developments in Geant4. Nucl Instrum Meth A 2016;835:186-225.

[25] Fernandez-Varea JM, Gonzalez-Munoz G, Galassi ME, Wiklund K, Lind BK, Ahnesjo A, et al. Limitations (and merits) of PENELOPE as a track-structure code. Int $\mathrm{J}$ Radiat Biol 2012;88:66-70.

[26] Bousis C, Emfietzoglou D, Hadjidoukas P, Nikjoo H. A Monte Carlo study of absorbed dose distributions in both the vapor and liquid phases of water by intermediate energy electrons based on different condensed-history transport schemes. Phys Med Biol 2008;53:3739-61.

[27] Lazarakis P, Incerti S, Ivanchenko V, Kyriakou I, Emfietzoglou D, Corde S, et al. Investigation of Track Structure and Condensed History physics models for applications in radiation dosimetry on a micro and nano scale in Geant4. Biom Phys Eng Express 2018;4:024001.

[28] Kyriakou I, Emfietzoglou D, Ivantchenko VN, Bordage MC, Guatelli S, Lazarakis P, et al. Microdosimetry of electrons in liquid water using the low-energy models of Geant4. J Appl Phys 2017;122:024303.

[29] Incerti S, Ivanchenko A, Karamitros M, Mantero A, Moretto P, Tran HN, et al. Comparison of GEANT4 very low energy cross section models with experimental data in water. Med Phys 2010;37:4692-708.

[30] Kyriakou I, Sefl M, Nourry V, Incerti S. The impact of new Geant4-DNA cross section models on electron track structure simulations in liquid water. J Appl Phys 2016;119:194902.

[31] Bordage MC, Bordes J, Edel S, Terrissol M, Franceries X, Bardies M, et al. Implementation of new physics models for low energy electrons in liquid water in Geant4-DNA. Phys Med: Eur J Med Phys 2016;32:1833-40.

[32] Cullen DE, Hubbell JH, Kissel L. EPDL97: The evaluated photon data library, 97 Version. Report No. UCRL-LR-50400-V6-R5. Lawrence Livermore National Laboratory, Livermore, 1997.

[33] Perkins ST, Cullen D, Chen M, Rathkopf J, Scofield J, Hubbell J. Tables and graphs of atomic subshell and relaxation data derived from the LLNL evaluated atomic data library (EADL), Z = 1-100. Report No. UCRL-50400-V30, Lawrence Livermore National Laboratory, Livermore, 1991.

[34] Perkins ST, Cullen D, Seltzer S. Tables and graphs of electron interaction crosssections from $10 \mathrm{eV}$ to $100 \mathrm{GeV}$ derived from the LLNL evaluated electron data library (EEDL), Z = 1-100. Report No. UCRL-50400-V31, Lawrence Livermore National Laboratory, Livermore, 1991.

[35] Seltzer SM. Cross sections for bremsstrahlung production and electron-impact ionization. In: Jenkins TM, Nelson WR, editors. Monte Carlo Transport of Electrons and Photons. New York: Plenum; 1988. p. 81-114.

[36] Ivanchenko VN, Kadri O, Maire M, Urban L. Geant4 models for simulation of multiple scattering. J Phys: Conf Ser 2010;219:032045.

[37] Incerti S, Ivanchenko V, Novak M. Recent progress of Geant4 electromagnetic physics for calorimeter simulation. J Instrum 2016;13. C02054.

[38] Liljequist D. A simple calculation of inelastic mean free path and stopping power for 50 eV-50 keV electrons in solids. J Phys D: Appl Phys 1983;16:1567.

[39] Incerti S, Kyriakou I, Bernal MA, Bordage MC, Francis Z, Guatelli S, et al. Geant4 DNA example applications for track structure simulations in liquid water: a report from the Geant4-DNA Project. Med Phys 2018;45:722-39.

[40] Emfietzoglou D, Moscovitch M. Inelastic collision characteristics of electrons in liquid water. Nucl Instrum Meth B 2002;193:71-8.

[41] Emfietzoglou D. Inelastic cross-sections for electron transport in liquid water: a comparison of dielectric models. Radiat Phys Chem 2003;66:373-85.

[42] Emfietzoglou D, Nikjoo H. The effect of model approximations on single-collision distributions of low-energy electrons in liquid water. Rad Res 2005;163:98-111.

[43] Kyriakou I, Incerti S, Francis Z. Improvements in Geant4 energy-loss model and the effect on low-energy electron transport in liquid water. Med Phys 2015;42:3870-6.

[44] Dingfelder M, Hantke D, Inokuti M, Paretzke HG. Electron inelastic scattering cross sections in liquid water. Radiat Phys Chem 1999;53:1-18.

[45] Bardies M, Kwok C, Sgouros G. Dose point-kernels for radionuclide dosimetry. In: Zaidi H, Sgouros G, editors. Therapeutic applications of Monte Carlo calculations in nuclear medicine. London: IOP Publishing Ltd; 2003. p. 158-74.

[46] Ferrer L, Chouin N, Bitar A, Lisbona A, Bardiès M. Implementing dosimetry in GATE: dose-point kernel validation with GEANT4 4.8.1. Cancer Biother Radiopharm 2007;22:125-9.

[47] ICRU, International Commission on Radiation Units and Measurements, Bethesda, MD, "Microdosimetry", Report 36; 1983.

[48] Zaider M, Brenner DJ. On the microdosimetric definition of quality factors. Radiat Res 1985;103:302-16.

[49] Lindborg L, Hultgvist M, Carlsson Tedgren A, Nikjoo H. Lineal energy and radiation quality in radiation therapy: model calculations and comparison with experiment. Phys Med Biol 2013;58:3089-105.

[50] Wuu CS, Zaider M. A calculation of the relative biological effectiveness of $125 \mathrm{I}$ and 103Pd brachytherapy sources using the concept of proximity function. Med Phys 1998;25:2186-9.

[51] Botta F, Mairani A, Dia A, Fasso A, Ferrari A, Ferrari M, et al. Calculation of electron and isotopes dose point kernels with FLUKA Monte Carlo code for dosimetry in nuclear medicine therapy. Med Phys 2011;38:3944-54. 